\title{
SOME PROPERTIES FOR CERTAIN ANALYTIC FUNCTIONS
}

\author{
D. Breaz, N. Breaz, S. Owa
}

Abstract. For analytic functions $f(z)$ in the open unit disk $U$ with $f(0)=0$ and $f^{\prime}(0)=1$, R. Singh and S. Singh (1982) showed some interesting results for univalency and starlikeness of $f(z)$. Also, for analytic functions $p(z)$ in $U$ with $p(0)=1$, M. Nunokawa gave some interesting theorems concerning with univalency and starlikeness of functions. The object of the present paper is to discuss some properties of functions which are the generalizations of results due to R. Singh and S. Singh, and due to M. Nunokawa.

2010 Mathematics Subject Classification: 30C45.

Keywords: analytic function, univalent, starlike, subordination

\section{INTRODUCTION}

Let $A$ be the class of functions $f(z)$ of the form

$$
f(z)=z+\sum_{n=2}^{\infty} a_{n} z^{n}
$$

which are analytic in the open unit disk $U=\{z \in \mathbb{C}:|z|<1\}$. A function $f(z)$ in the class $A$ is said to be univalent in $U$ if it satisfies $f\left(z_{1}\right) \neq f\left(z_{2}\right)$ for $z_{1} \neq z_{2}\left(z_{1} \in\right.$ $\left.U, z_{2} \in U\right)$ and denoted by $f(z) \in S$. Also a function $f(z) \in S$ is said to be starlike in $U$ if $f(z)$ maps $U$ to a starlike domain with respect to the origin. We write that $f(z) \in S^{*}$ for such functions. It is well-known that $f(z) \in S^{*}$ is equivalent to

$$
\operatorname{Re}\left(\frac{z f^{\prime}(z)}{f(z)}\right)>0 \quad(z \in U) .
$$

Let $P_{0}(a)$ denote the class of functions $p(z)$ with $p(0)>0$ which satisfy

$$
|p(z)-p(0)|<a \quad(z \in U)
$$

for some real $a(0<a \leq p(0))$.

In order to discuss our problems for $p(z) \in P_{0}(a)$, we have to recall here the following results. 
Lemma 1. (I.S. Jack [2]) Let $w(z)$ be analytic in $U$ with $w(0)=0$. If $|w(z)|$ attains its maximum value on the circle $|z|=r<1$ at a point $z_{0}$, then

$$
z_{0} w^{\prime}\left(z_{0}\right)=k w\left(z_{0}\right) \quad(k \geq 1) .
$$

Lemma 2. (M. Nunokawa [4]) Let $p(z)$ be analytic in $U, p(0)=1$ and suppose that there exists a point $z_{0} \in U$ such that

$$
\begin{gathered}
\operatorname{Re} p(z)>0 \quad \text { for } \quad|z|<\left|z_{0}\right|<1, \\
\operatorname{Re} p\left(z_{0}\right)=0 \quad \text { and } \quad p\left(z_{0}\right)= \pm i a .
\end{gathered}
$$

Then

$$
\frac{z_{0} p^{\prime}\left(z_{0}\right)}{p\left(z_{0}\right)}=i k
$$

where

$$
k \geq \frac{1}{2}\left(a+\frac{1}{a}\right) \geq 1 \quad \text { when } \quad p\left(z_{0}\right)=i a \quad(a>0)
$$

and

$$
k \leq-\frac{1}{2}\left(a+\frac{1}{a}\right) \leq-1 \quad \text { when } \quad p\left(z_{0}\right)=-i a \quad(a>0)
$$

Further, we need

Lemma 3. (M. Nunokawa [5]) Let $p(z)$ be analytic in $U, p(0)=1, p(z) \neq 0$ in $U$ and suppose that there exists a point $z_{0} \in U$ such that

$$
|\arg (p(z))|<\frac{\pi}{2} \alpha \quad \text { for } \quad|z|<\left|z_{0}\right|<1,
$$

and

$$
\left|\arg \left(p\left(z_{0}\right)\right)\right|=\frac{\pi}{2} \alpha
$$

for $\alpha>0$. Then

$$
\frac{z_{0} p^{\prime}\left(z_{0}\right)}{p\left(z_{0}\right)}=i k \alpha
$$

where

$$
\begin{aligned}
& k \geq \frac{1}{2}\left(a+\frac{1}{a}\right) \geq 1 \quad \text { when } \arg p\left(z_{0}\right)=\frac{\pi}{2} \alpha, \\
& k \leq-\frac{1}{2}\left(a+\frac{1}{a}\right) \leq-1 \quad \text { when } \quad \arg p\left(z_{0}\right)=-\frac{\pi}{2} \alpha,
\end{aligned}
$$

and

$$
p\left(z_{0}\right)^{\frac{1}{\alpha}}= \pm i a \quad(a>0) .
$$




\section{SOME PROPERTIES}

We first introduce the following two results concerning with our results.

Theorem A. (R. Singh and S. Singh [7]) If $f(z) \in A$ satisfies

$$
\left|f^{\prime}(z)-1\right|^{1-\gamma}\left|z f^{\prime \prime}(z)\right|^{\gamma}<1 \quad(z \in U)
$$

for some real $\gamma \geq 0$, then

$$
\left|f^{\prime}(z)-1\right|<1 \quad(z \in U) .
$$

Theorem B. (M. Nunokawa [6]) Let $p(z)$ be analytic in $U$ with $p(0)=1$. If $p(z)$ satisfies

$$
\left|p(z)+z p^{\prime}(z)-1\right|<\sqrt{2} \quad(z \in U)
$$

then

$$
|p(z)-1|<\frac{\sqrt{2}}{2} \quad(z \in U)
$$

and

$$
|\arg (p(z))|<\frac{\pi}{4} \quad(z \in U)
$$

Our first property for $P_{0}(a)$ is as follows.

Theorem 4. Let $p(z)$ be analytic in $U$ with $p(0)>0$. If $p(z)$ satisfies

$$
|p(z)-p(0)|^{\alpha}\left|z p^{\prime}(z)\right|^{\beta}<a^{\alpha+\beta} \quad(z \in U)
$$

for some $\alpha \geq 0, \beta \geq 0$, and $0<a \leq p(0)$, then $p(z) \in P_{0}(a)$ and

$$
|\arg (p(z))|<\sin ^{-1}\left(\frac{a}{p(0)}\right) \quad(z \in U) .
$$

Proof. Let us define a function $w(z)$ by

$$
w(z)=\frac{p(z)-p(0)}{a} \quad(z \in U)
$$

with $w(0)=0$. Since $z p^{\prime}(z)=a z w^{\prime}(z)$, we see that

$$
|p(z)-p(0)|^{\alpha}\left|z p^{\prime}(z)\right|^{\beta}=|a w(z)|^{\alpha}\left|a z w^{\prime}(z)\right|^{\beta}=a^{\alpha+\beta}|w(z)|^{\alpha-\beta}\left|\frac{z w^{\prime}(z)}{w(z)}\right|^{\beta} .
$$


We suppose that there exists a point $z_{0} \in U$ such that

$$
\max _{|z| \leq\left|z_{0}\right|}|w(z)|=\left|w\left(z_{0}\right)\right|=1
$$

then Lemma 1 gives us that $w\left(z_{0}\right)=e^{i \theta}$ and $z_{0} w^{\prime}\left(z_{0}\right)=k w\left(z_{0}\right)(k \geq 1)$. This shows us that

$$
\left|p\left(z_{0}\right)-p(0)\right|^{\alpha}\left|z_{0} p^{\prime}\left(z_{0}\right)\right|^{\beta}=a^{\alpha+\beta} k^{\beta} \geq a^{\alpha+\beta}
$$

which contradicts our condition (10) for $p(z)$. Therefore we say that there is no $z_{0} \in U$ such that $\left|w\left(z_{0}\right)\right|=1$. This means that $|w(z)|<1$ for all $z \in U$, that is, that

$$
|p(z)-p(0)|<a \quad(z \in U) .
$$

Thus we obtain that $p(z) \in P_{0}(a)$ and

$$
|\arg (p(z))|<\sin ^{-1}\left(\frac{a}{p(0)}\right) \quad(z \in U) .
$$

Letting $p(z)=f^{\prime}(z)$ for $f(z) \in A$ in Theorem 4 , we see

Corollary 5. If $f(z) \in A$ satisfies

$$
\left|f^{\prime}(z)-1\right|^{\alpha}\left|z f^{\prime \prime}(z)\right|^{\beta}<a^{\alpha+\beta} \quad(z \in U)
$$

for some $\alpha \geq 0, \beta \geq 0$, and $0<a \leq 1$, then we have

$$
\left|f^{\prime}(z)-1\right|<a \quad(z \in U)
$$

and

$$
\left|\arg \left(f^{\prime}(z)\right)\right|<\sin ^{-1} a \quad(z \in U) .
$$

Corollary 5 is the generalization of Theorem A.

Next, we consider

Theorem 6. Let $p(z)$ be analytic in $U$ with $p(0)>0$. If $p(z)$ satisfies

$$
\left|p(z)+b z p^{\prime}(z)-p(0)\right|<a(1+b) \quad(z \in U)
$$

for some $a(0<a \leq p(0))$ and $b>0$, then we have $p(z) \in P_{0}(a)$ and

$$
|\arg (p(z))|<\sin ^{-1}\left(\frac{a}{p(0)}\right) \quad(z \in U) .
$$


Proof. Defining a function $w(z)$ by (12), we obtain that

$$
\left|p(z)+b z p^{\prime}(z)-p(0)\right|=\left|a w(z)+a b z w^{\prime}(z)\right|=a|w(z)|\left|1+b \frac{z w^{\prime}(z)}{w(z)}\right| .
$$

Therefore, we suppose that there exists a point $z_{0} \in U$ such that

$$
\max _{|z| \leq\left|z_{0}\right|}|w(z)|=\left|w\left(z_{0}\right)\right|=1
$$

Then Lemma 1 gives us that $w\left(z_{0}\right)=e^{i \theta}$ and

$$
\frac{z_{0} w^{\prime}\left(z_{0}\right)}{w\left(z_{0}\right)}=k \geq 1
$$

It follows from the above that

$$
\left|p\left(z_{0}\right)+b z_{0} p^{\prime}\left(z_{0}\right)-p(0)\right|=a|1+b k| \geq a(1+b) .
$$

This contradicts the condition (18) of the theorem. Therefore $w(z)$ has to satisfy that

$$
|w(z)|<1 \quad(z \in U) .
$$

This completes the proof of the theorem.

Letting $a=\frac{1}{\sqrt{2}}$ in Theorem 6 , we have

Corollary 7. Let $p(z)$ be analytic in $U$ with $p(0)=1$. If $p(z)$ satisfies

$$
\left|p(z)+b z p^{\prime}(z)-1\right|<\frac{1+b}{\sqrt{2}} \quad(z \in U)
$$

for some $b>0$, then we have

$$
|p(z)-1|<\frac{1}{\sqrt{2}} \quad(z \in U)
$$

and

$$
|\arg (p(z))|<\frac{\pi}{4} \quad(z \in U)
$$


Corollary 7 is the generalization of Theorem B.

Next, we introduce the following result due to R. Singh and S. Singh [7].

Theorem C. If $f(z) \in A$ satisfies

$$
\left|f^{\prime}(z)-1\right|^{1-\gamma}\left|1+\frac{z f^{\prime \prime}(z)}{f^{\prime}(z)}\right|^{\gamma}<\left(\frac{3}{2}\right)^{\gamma} \quad(z \in U)
$$

for some real $\gamma \geq 0$, then

$$
\left|f^{\prime}(z)-1\right|<1 \quad(z \in U) .
$$

For Theorem C, we derive

Theorem 8. Let $p(z)$ be analytic in $U$ with $p(0)>0$. If $p(z)$ satisfies

$$
|p(z)-p(0)|^{\alpha}\left|1+\frac{z p^{\prime}(z)}{p(z)}\right|^{\beta}<a^{\alpha}\left(1+\frac{a}{a+p(0)}\right)^{\beta} \quad(z \in U)
$$

for some real $\alpha \geq 0, \beta \geq 0$ and $\frac{p(0)}{\sqrt{2}} \leq a \leq p(0)$, then we have $p(z) \in P_{0}(a)$ and

$$
|\arg (p(z))|<\sin ^{-1}\left(\frac{a}{p(0)}\right) \quad(z \in U) .
$$

Proof. Defining the function $w(z)$ by (12), we obtain that

$$
\frac{z p^{\prime}(z)}{p(z)}=\frac{a z w^{\prime}(z)}{a w(z)+p(0)} \text {. }
$$

It follows from (12) and (27) that

$$
\begin{array}{r}
|p(z)-p(0)|^{\alpha}\left|1+\frac{z p^{\prime}(z)}{p(z)}\right|^{\beta}=a^{\alpha}|w(z)|^{\alpha}\left|1+\frac{a z w^{\prime}(z)}{a w(z)+p(0)}\right|^{\beta} \\
=a^{\alpha}|w(z)|^{\alpha+\beta}\left|\frac{1}{w(z)}+\frac{a \frac{z w^{\prime}(z)}{w(z)}}{a w(z)+p(0)}\right|^{\beta} .
\end{array}
$$

Therefore, we suppose that there exists a point $z_{0} \in U$ such that

$$
\max _{|z| \leq\left|z_{0}\right|}|w(z)|=\left|w\left(z_{0}\right)\right|=1 .
$$


Applying Lemma 1 for $w(z)$, we say that $w\left(z_{0}\right)=e^{i \theta}$ and

$$
\frac{z_{0} w^{\prime}\left(z_{0}\right)}{w\left(z_{0}\right)}=k \geq 1
$$

Thus we see that

$$
\begin{gathered}
\left|p\left(z_{0}\right)-p(0)\right|^{\alpha}\left|1+\frac{z_{0} p^{\prime}\left(z_{0}\right)}{p\left(z_{0}\right)}\right|^{\beta} \\
=a^{\alpha}\left|e^{-i \theta}+\frac{a k}{a e^{i \theta}+p(0)}\right|^{\beta} \geq a^{\alpha}\left(\frac{a^{2}(k+1)^{2}+p^{2}(0)+2 a(k+1) p(0) \cos \theta}{a^{2}+p^{2}(0)+2 a p(0) \cos \theta}\right)^{\beta} \\
\geq a^{\alpha}\left(1+\frac{a k}{a+p(0)}\right)^{\beta} \geq a^{\alpha}\left(1+\frac{a}{a+p(0)}\right)^{\beta} .
\end{gathered}
$$

Since this contradicts our condition (26) we say that $|w(z)|<1(z \in U)$. This completes the proof of the theorem.

Letting $p(z)=f^{\prime}(z)$ for $f(z) \in A$ in Theorem 8, we have

Corollary 9. If $f(z) \in A$ satisfies

$$
\left|f^{\prime}(z)-1\right|^{\alpha}\left|1+\frac{z f^{\prime \prime}(z)}{f^{\prime}(z)}\right|^{\beta}<a^{\alpha}\left(\frac{2 a+1}{a+1}\right)^{\beta} \quad(z \in U)
$$

for some real $\alpha \geq 0, \beta \geq 0$, and $\frac{1}{\sqrt{2}} \leq a \leq 1$, then

$$
\left|f^{\prime}(z)-1\right|<a \quad(z \in U) .
$$

Corollary 3 is the generalization of Theorem C.

\section{Subordinations of FUnCtions}

Let $f(z)$ and $g(z)$ be analytic in $U$. Then the function $f(z)$ is said to be subordinated to $g(z)$ if there exists a function $w(z)$ which is analytic in $U$ with $w(0)=0$ and $|w(z)|<1$, and such that $f(z)=g(w(z))$. We denote this subordination by

$$
f(z) \prec g(z) \quad(z \in U) .
$$


If $g(z)$ is univalent in $U$, then the subordination $f(z) \prec g(z)(z \in U)$ is equivalent to $f(0)=g(0)$ and $f(U) \subset g(U)$ (see [1], [3]).

Now we derive

Theorem 10. Let a function $p(z)$ be analytic in $U$ with $p(0)=1$. If $p(z)$ satisfies

$$
\operatorname{Re}\left(p(z)+\frac{z p^{\prime}(z)}{p(z)}\right)< \begin{cases}\frac{5 \alpha-1}{2(\alpha+1)} & (1<\alpha \leq 2) \\ \frac{\alpha+1}{2(\alpha-1)} & (2<\alpha<3)\end{cases}
$$

for $z \in U$, then

$$
p(z) \prec \frac{\alpha(1-z)}{\alpha-z} \quad(z \in U)
$$

and

$$
\left|p(z)-\frac{\alpha}{\alpha+1}\right|<\frac{\alpha}{\alpha+1} \quad(z \in U)
$$

Proof. Let us define $w(z)$ by

$$
p(z)=\frac{\alpha(1-w(z))}{\alpha-w(z)} \quad(z \in U) .
$$

Then we obtain that

$$
p(z)+\frac{z p^{\prime}(z)}{p(z)}=\frac{\alpha(1-w(z))}{\alpha-w(z)}-\frac{z w^{\prime}(z)}{1-w(z)}+\frac{z w^{\prime}(z)}{\alpha-w(z)} .
$$

Suppose that there exists a point $z_{0} \in U$ such that

$$
\max _{|z| \leq\left|z_{0}\right|}|w(z)|=\left|w\left(z_{0}\right)\right|=1 .
$$

Therefore, Lemma 1 gives us that $w\left(z_{0}\right)=e^{i \theta}$ and

$$
\frac{z_{0} w^{\prime}\left(z_{0}\right)}{w\left(z_{0}\right)}=k \geq 1
$$


It follows from the above that

$$
\begin{aligned}
& \operatorname{Re}\left(p\left(z_{0}\right)+\frac{z_{0} p^{\prime}\left(z_{0}\right)}{p\left(z_{0}\right)}\right)=\operatorname{Re}\left(\frac{\alpha\left(1-e^{i \theta}\right)}{\alpha-e^{i \theta}}-\frac{k e^{i \theta}}{1-e^{i \theta}}+\frac{k e^{i \theta}}{\alpha-e^{i \theta}}\right) \\
& =\frac{\alpha(\alpha+1)(1-\cos \theta)}{\alpha^{2}+1-2 \alpha \cos \theta}+\frac{k}{2}+\frac{k(\alpha \cos \theta-1)}{\alpha^{2}+1-2 \alpha \cos \theta}=\frac{\alpha+1}{2}+\frac{\left(\alpha^{2}-1\right)(k+1-\alpha)}{2\left(\alpha^{2}+1-2 \alpha \cos \theta\right)} \\
& \geq \frac{\alpha+1}{2}+\frac{\left(\alpha^{2}-1\right)(2-\alpha)}{2\left(\alpha^{2}+1-2 \alpha \cos \theta\right)} \\
& \geq \begin{cases}\frac{\alpha+1}{2}+\frac{(\alpha-1)(2-\alpha)}{2(\alpha+1)}=\frac{5 \alpha-1}{2(\alpha+1)} & (1<\alpha \leq 2) \\
\frac{\alpha+1}{2}+\frac{(\alpha+1)(2-\alpha)}{2(\alpha-1)}=\frac{\alpha+1}{2(\alpha-1)} & (2<\alpha<3) .\end{cases}
\end{aligned}
$$

This contradicts the condition (34) of the theorem. This means that there is no any point $z_{0} \in U$ such that $\left|w\left(z_{0}\right)\right|=1$ and $w(0)=0$. It follows that $|w(z)|<1$ for all $z \in U$. Therefore we say that

$$
p(z) \prec \frac{\alpha(1-z)}{\alpha-z} \quad(z \in U) .
$$

Further, nothing that

$$
|w(z)|=\left|\frac{\alpha(p(z)-1)}{p(z)-\alpha}\right|<1 \quad(z \in U),
$$

we obtain (36). This completes the proof of the theorem.

Taking $p(z)=\frac{z f^{\prime}(z)}{f(z)}$ in Theorem 10, we may have

Corollary 11. If $f(z) \in A$ satisfies

$$
\operatorname{Re}\left(1+\frac{z f^{\prime \prime}(z)}{f^{\prime}(z)}\right)< \begin{cases}\frac{5 \alpha-1}{2(\alpha+1)} & (1<\alpha \leq 2) \\ \frac{\alpha+1}{2(\alpha-1)} & (2<\alpha<3)\end{cases}
$$

for $z \in U$, then we have

$$
\frac{z f^{\prime}(z)}{f(z)} \prec \frac{\alpha(1-z)}{\alpha-z} \quad(z \in U)
$$

and

$$
\left|\frac{z f^{\prime}(z)}{f(z)}-\frac{\alpha}{\alpha+1}\right|<\frac{\alpha}{\alpha+1} \quad(z \in U) .
$$


Remark 1. The inequality shows that $f(z) \in S^{*}$ and the function

$$
f(z)=z\left(1-\frac{z}{\alpha}\right)^{\alpha-1} \quad(1<\alpha<3)
$$

is the extremal function for Theorem 10.

Remark 2. R. Singh and S. Singh in 1982 showed that if $f(z) \in A$ satisfies

$$
\frac{z f^{\prime}(z)}{f(z)} \prec \frac{2(1-z)}{1-z} \quad(z \in U)
$$

then $f(z) \in S^{*}$. Thus Corollary 11 is the improvement of the result due to $R$. Singh and S. Singh [7].

\section{REFERENCES}

[1] P.L. Duren, Univalent Functions, Springer Verlag, N.Y., Berlin, Heidelberg, Tokyo, (1983).

[2] I.S. Jack, Functions starlike and convex of order $\alpha$, J. London Math. Soc. 3(1971), 469- 474.

[3] S.S. Miller and P.T. Mocanu, Differential subordinations, Theory and Applications, Marcel Dekker, New York, Basel (2000).

[4] M. Nunokawa, On properties of non-Carathéodory functions, Proc. Japan Acad. 68 (1992), 152-153.

[5] M. Nunokawa, On the order of strongly starlikeness of strongly convex functions, Proc. Japan Acad. 69 (1993), 234-237.

[6] M. Nunokawa, A class of multivalent functions, Tsukuba J. Math. 16 (1992), 423-428.

[7] R. Singh and S. Singh, Some sufficient conditions for univalence and starlikeness, Coll. Math. 47 (1982), 309-314.

Daniel Breaz,

Department of Mathematics-Informatics, Faculty of Sciences,

"1 Decembrie 1918" University of Alba Iulia,

N. Iorga St., No. 11-13, 510009 Alba Iulia, România.

email:dbreaz@uab.ro 
Nicoleta Breaz,

Department of Mathematics-Informatics,

Faculty of Sciences,

"1 Decembrie 1918" University of Alba Iulia,

N. Iorga St., No. 11-13, 510009 Alba Iulia, România.

email: nbreaz@uab.ro

Shigeyoshi Owa,

Honorary professor

"1 Decembrie 1918" University of Alba Iulia,

N. Iorga St., No. 11-13, 510009 Alba Iulia, România.

email: shige21@ican.zaq.ne.jp 